\title{
Frequency of Side Effects after First Dose of Vaccination against COVID-19 Among the People of Bangladesh
}

\author{
H. M. Khaleduzzaman and Nafisa Jabin Mishu
}

\section{ABSTRACT}

\begin{abstract}
Vaccine is an important means to prevent COVID-19. It is a new modality of treatment for COVID-19 so many more to know about it especially in case of Bangladeshi people. Total $18.1 \%$ volunteers out of 1160 participated were noticed various side effects. These effects have been found within two months of observation following first dosage of vaccination against COVID-19. Fortunately, most of the side effects were mild and within 48 hours of vaccination. Its effectiveness is still questionable. To prove effectiveness, safety, side effects and additional benefit needs further study.
\end{abstract}

Keywords: COVID-19; vaccine; side effect.

\author{
Submitted : April 25, 2021 \\ Published : May 17, 2021 \\ ISSN: $2593-8339$ \\ DOI: $10.24018 /$ ejmed.2021.3.3.836 \\ Dr H. M. Khaleduzzaman* \\ MBBS, FCPS (medicine) Major, Bangladesh \\ Army, Bangladesh. \\ (e-mail: raju47cmc@gmail.com) \\ Dr Nafisa Jabin Mishu \\ MBBS, M-Phil (Microbiology) Dept of \\ Microbiology, Dhaka Medical College, Dhaka, \\ Bangladesh. \\ (e-mail: jabinjabin560@gmail.com) \\ *Corresponding Author
}

\section{INTRODUCTION}

COVID-19 is a zoonotic disease caused by SARS-COV2. [1] It is an enveloped virus [2]. In 2020 it out broke as a pandemic and whole word have experienced its severity [3]. There is no definitive treatment yet for this disease but fortunately our scientists have invented vaccine against it. Vaccine is a process by which body become familiarize to certain antigen of specific organism. Different vaccines have developed to prevent COVID-19, The Oxford/AstraZeneca COVID-19 vaccine has already got approval from WHO and in Bangladesh this vaccine is giving to priority based people. This vaccine has already undergone many studies before experiencing to human but none of them among Bangladeshi people. So, i intended to do a research on this vaccine especially on side effects of this vaccine on Bangladeshi people. Vaccine introduce body to certain component of organisms and enhance natural immunity, both cell mediated and antibody mediated immunity and prepare body to defend against specific viruses and bacteria.

Vaccine is an essential tool to fight against COVID-19 and it is hugely encouraging to see so many vaccines proving successful trial and many more on the process of development. Scientists from all over the world are innovating to bring new tests, treatments and vaccines that will collectively save lives and hope ultimately this pandemic will be the end. As vaccine against COVID-19 is new so many more studies are required to establish its final outcome, side effects, efficacy and safety.

\section{MATERIALS AND METHODS}

A cross-sectional observational study was carried out with a sample of 1160 . They underwent vaccination against COVID-19 in different centers of Bangladesh. Vaccinated individual had been observed for two months after vaccination whether any side effects occur or not. For this purpose, a Google form was generated and the link of structured questioner (https://docs.google.com/forms/d/1pZa86L8tbTp6CHa19bF BHDrgIHxLTUklk8Ymg6_fG_U/edit?vc $=0 \& \mathrm{c}=0 \& \mathrm{w}=1 \& \mathrm{fl} \mathrm{r}$ $=0)$ was given to every individual along with my contact number to inform any side effects noticed by vaccinated individual. For this study a written informed consent was taken to participate in this study. No monitory benefit was given to participants. It was totally involuntary and if anybody wants to withdraw him or her from this study, there was total flexibility. The sample was collected randomly who were attended at different vaccination centers across the country including both civil and uniform personnel who have fulfilled the inclusion and exclusion criteria. Vaccinated individual are allowed to wait 30 minutes at vaccination center to observe any immediate serious side effects and all vaccination center were well equipped to manage immediate serious side effects including vasopressors, I/V fluids, Oxygen's and emergency resuscitation facilities. The study variables were included common side effects observed in other studies but options are kept to add new or additional side effects other than structured one. Data were collected by purposive simple random sampling method with a structured 
questionnaire. Finally, data analysis was done using SPSS25.

Individual who have vaccinated against COVID-19 and those who have given informed consent were included in this study. One the other hand who were suffering from HIV or who were sever immune compromised, pregnant, lactating mother were excluded from this study. Participants who were taking chronic steroid due to any reason, who were unwilling to participate and age bellow 18 years of age were out of this study. Following participants were included in this study like health workers who were at high risk of becoming infected by SARS-COV-2, employment groups who were at elevated risk of transmitting infection because they are unable to effectively physically distance, whose age 40 years or more, all members of Ministry of health and welfare, all government and non-government service personnel related to health service, freedom fighters, Armed forces personnel, private front line workers related to law enforcing agency, media workers, person related to burial, persons related to emergency service like-water supply, electricity, gas, fireservice, sewage disposal, employees working at rail station, airport, bus station, bank worker, overseas worker and players of national team.

\section{RESULTS}

Total 1160 individuals who were vaccinated against COVID-19 participate voluntarily in this study. Participants were from 26 to 60 years age group and $95 \%$ of them were male while only $5 \%$ were female showed in Fig. 1 . Most of the participants were married $(96 \%)$ though $4 \%$ were unmarried showed in Fig. 2. Most of participants $(81.9 \%)$ did not notice any side effects but $18.1 \%$ had been suffering from side effects of various modalities following COVID-19 vaccination (Fig. 3). They noticed side effects mostly (45\%) on first and second day showed in Fig. 4. In this study most of the side effects are mild like fever $(16.8 \%)$, headache $(16.8 \%)$, sore arm $(11.1 \%)$, joint pain $(10.3 \%)$, fatigue $7.2 \%$, chills $(6.3 \%)$ and transient skin rash $(0.9 \%)$ but some serious side effects were also found like seizure (1.6\%) and unconsciousness $(1.6 \%)$ whose required hospitalization though they regain consciousness without any neurological deficit. They return to home after 24 hours of hospital supervision. No anaphylactic shock was noticed among the participants. Following chronic diseases were noticed among COVID-19 vaccinated participants like diabetes mellitus (7.4\%), Hypertension (2.6\%), chronic liver disease $(1.7 \%)$, chronic obstructive pulmonary disease $(0.5 \%)$, Ischemic heart disease with hypertension $(0.3 \%)$ and Asthma $(0.1 \%)$ showed in Fig. 6.

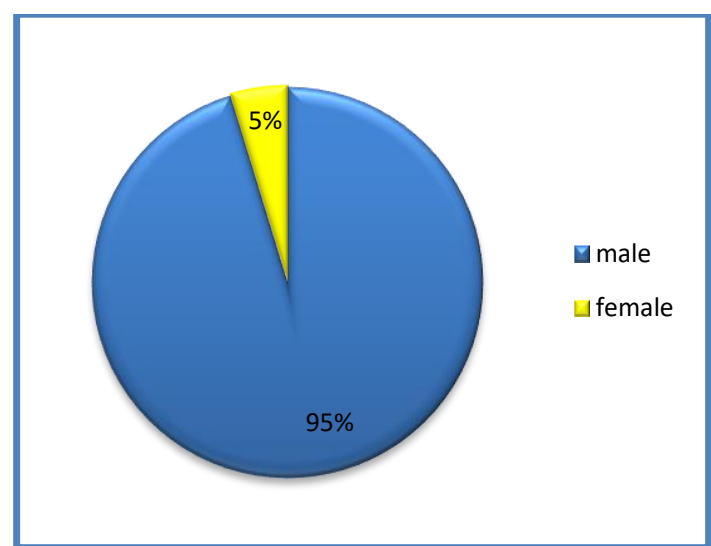

Fig. 1. Sex distribution among the respondents.

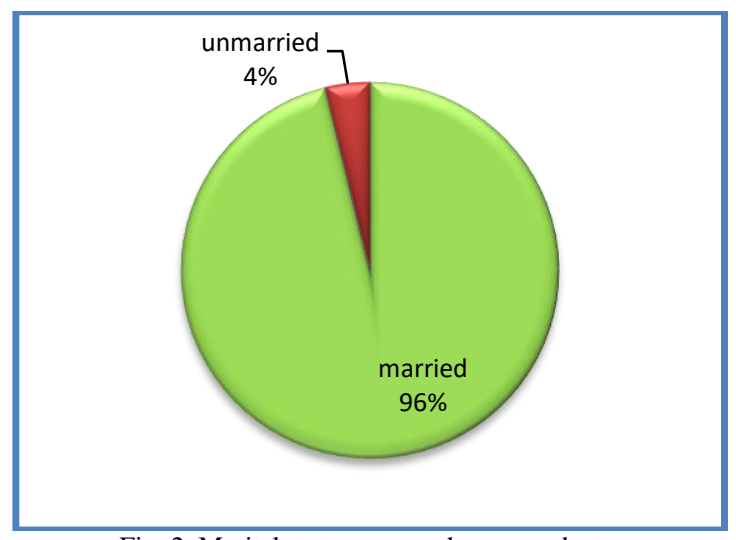

Fig. 2. Marital status among the respondents.

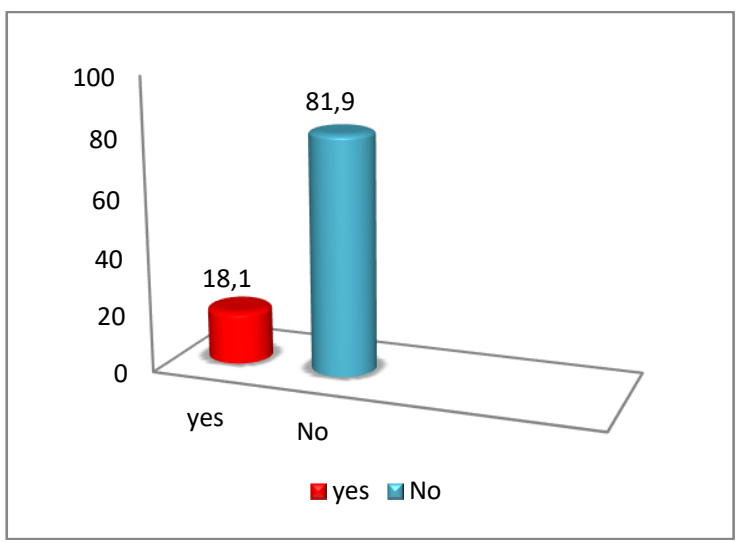

Fig. 3. Distributions of side effects among the respondents.

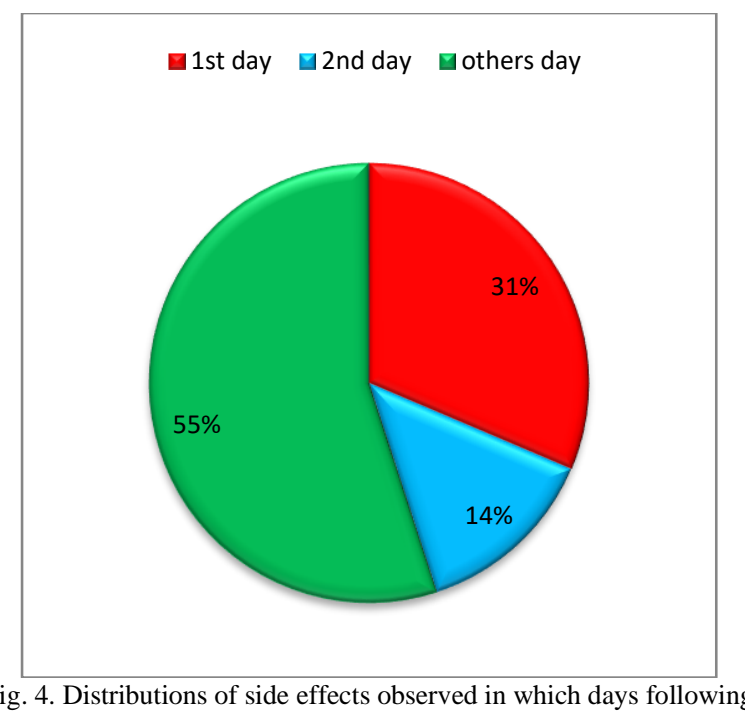

Fig. 4. Distributions of side effects observed in which days following vaccination? 


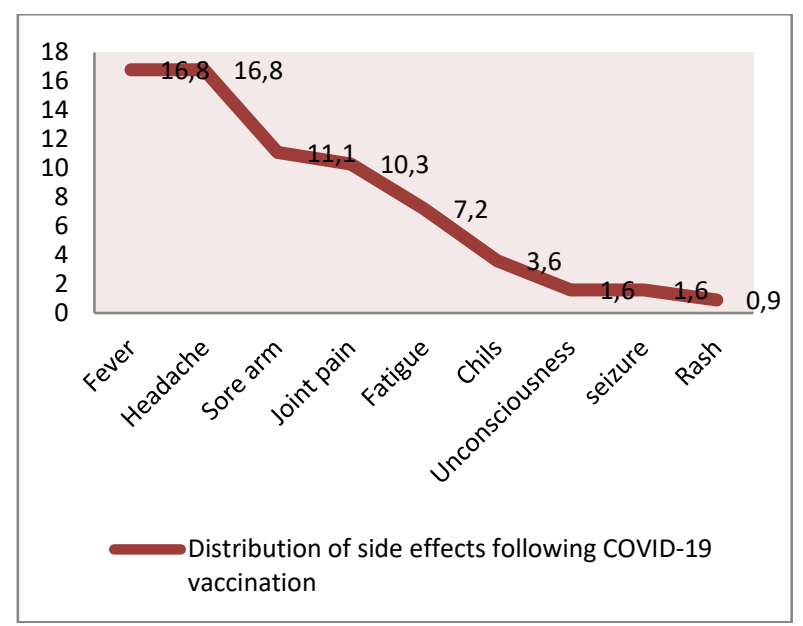

Fig. 5. Distributions of side effects observed following COVID-19 vaccination.

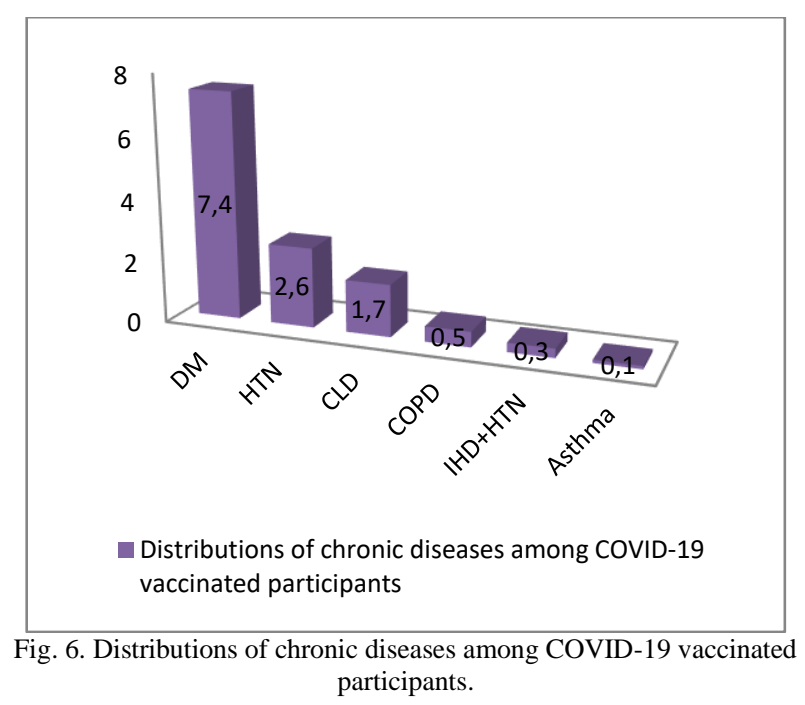

\section{DISCUSSION}

In this study most common side effects are fever (16.8\%), and headache $(16.8 \%)$, other side effects were sore arm $(11.1 \%)$, joint pain $(10.3 \%)$, fatigue $7.2 \%$, chills $(6.3 \%)$ and transient skin rash $(0.9 \%)$. There were observed some serious side effects like seizure (1.6\%) and unconsciousness (1.6\%). These findings slightly differ with other study where most common side effects were soreness at injection sites, but similarity was found with other findings [4]. Side effects differ from vaccine to vaccine and from individual to individual. In case of Johnson \& Johnson vaccine higher prevalence of side effects like headache (39\%), fatigue $38 \%$ ), muscle pain $33 \%$, nausea $14 \%$ and fever $10 \%$ but only $25 \%$ side effects noticed who took Moderna or Pfizer-BioNTech vaccine. [5] In this study, 45\% side effects occur on first and second day which is similar with other study [4]. Here we observed two months after vaccination, but no delayed side effects were noticed but some study found delayed side effects two months after vaccination [6].

\section{CONCLUSION}

Vaccine is an effective means to prevent any infection. COVID-19 still showing its devastating effects on world but we have to fight against it. Effective and safe vaccine can bring the freedom from SARS COV-2. To prove safe and effective vaccine many more study on this vaccine is required. Though vaccine is necessary tools, it has some side effects and so far, no life threatening side effects observed in Bangladeshi people.

\section{REFERENCES}

[1] P. R.-O. Y. O. B. A. M.-B. L. E. M. J. T. Y.-M. R. A. K. L. M. J. R. T D. M. Najmul Haider, "Najmul Haider,1 Peregrine Rothman-Ostrow,2 Abdinasir Yusuf Osman,1 Liã Bárbara Arruda,3 Laura MacfarlaneBerry,4 Linzy Elton,3 Margaret J. Thomason,3 Dorothy YeboahManu,5 Rashid Ansumana,6 Nathan Kapata,7 Leonard Mboera,8 Jonathan Rushton,2 Timothy D. Mc," Front Public Health. , p. 8, 2020.

[2] M. Marlene L. Durand, "Novel Coronavirus Infection," New england college of optometry, p. 4, 2020.

[3] A. O. O. J. O. M. \&. R. T. K. Kamoru A. Adedokun, "A close look at the biology of SARS-CoV-2, and the potential influence of weather conditions and seasons on COVID-19 case spread," Infectious Diseases of Poverty, p. 1, 2020.

[4] W.MOSS, "Side effects of COVID-19 vaccines," COVID-19SCHOOL OF PUBLIC HEALTH EXPERT INSIGHTS, pp. 1-2, 2020.

[5] Michelle Crouch and Rachel Nania, " "What Are the Side Effects of COVID-19 Vaccines?," " HEALTH Conditions \& Treatments, USA, , pp. 1-2, 2021.

[6] P. PACK, " "Feature Article: Long-term Side Effects of COVID-19 Vaccine? What We Know," children's hospital of philadelphia, philadelphia, p. 1, 2021.

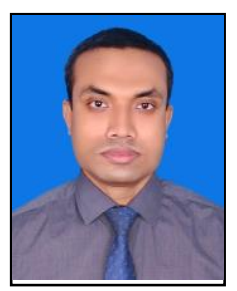

Dr H. M. Khaleduzzaman, MBBS, FCPS (medicine) Major, Bangladesh Army, Bangladesh. Place of birth: Jashore, Bangladesh Date of birth: 31 December 1987.

Educational background: MBBS from Chittagong Medical College, Chittagong Bangladesh, in the year 2010, FCPS in Medicine from Bangladesh College of Physicians and surgeon, Dhaka Bangladesh, in the year 2021, Major field of study is internal medicine.

$\mathrm{He}$ is a military person presently working as a major. Work experience is 9 years in the field of medicine. Currently, he is working in Border Guard Bangladesh as a deputy director and medicine specialist. His Previous publications were:

1. Prevalence and Associated Risk Factors of Hypertension: A CrossSectional Study in Aguelhok which was published at the Grant Medical Journal.

2. Frequency of Hypertension after 10 Years of Military Services in Bangladesh Army which was published at European Journal of Medical and Health Sciences.

His hobbies are passing time with family and reading books. 